\title{
Reliability and validity of the Myanmar version of the child oral health impact profile - short form 19
}

\author{
Zar Chi Kyaw Myint ${ }^{1,2}$; Takashi Zaitsu ${ }^{1 *}$; Akiko Oshiro ${ }^{1}$ Ko Ko Soe ${ }^{2}$;oko Kawaguchi ${ }^{1}$ \\ ${ }^{1}$ Department of Oral Health Promotion, Tokyo Medical and Dental University, Japan \\ ${ }^{2}$ Department of Preventive and Community Dentistry, University of Dental Medicine (Yangon), Myanmar
}

\author{
*Corresponding Author(s): Takashi Zaitsu \\ Department of Oral Health Promotion, Tokyo \\ Medical and Dental University, 1-5-45 Yushima. \\ Bunkyo-ku, Tokyo 113-8549, Japan \\ Tel: +81-3-5803-5476, Fax: +81-3-5803-0194; \\ Email: zaitsu.ohp@tmd.ac.jp
}

Received: Mar 26, 2020

Accepted: Apr 29, 2020

Published Online: May 05, 2020

Journal: Annals of Epidemiology and Public health

Publisher: MedDocs Publishers LLC

Online edition: http://meddocsonline.org/

Copyright: (C) Zaitsu T (2020). This Article is

distributed under the terms of Creative Commons

Attribution 4.0 International License

Keywords: Quality of life; Oral health status; Reliability; Validity; Myanmar; COHIP-SF 19.

\section{Abstract}

Objective: The original English version of Child Oral Health Impact Profile-Short Form 19 (COHIP-SF 19), used to evaluate the oral health-related quality of life, has been translated into Chinese, Arabic, and Portuguese. The aim of this study was to assess the psychometric properties of a Myanmar version of the COHIP-SF 19 (Myanmar COHIP-SF 19) and to identify the explanatory factors that impact the COHIP-SF 19.

Methods: The Myanmar COHIP-SF 19 was developed by a standard forward-backward translation. In total, 537 fifthgrade school children, aged $10-11$ years ( 251 boys and 286 girls), completed the final version of the instrument and underwent oral examination. Psychometric properties such as internal consistency, test-retest reliability, and concurrent and discriminant validity were tested.

Results: The Cronbach's alpha value for the total COHIPSF 19 showed good internal consistency: the Cronbach's alpha value was 0.81 and intraclass correlation coefficient was 0.86 . These results are consistent with those of the original English COHIP-SF 19. The concurrent validity test demonstrated higher total COHIP-SF 19 scores and higher scores on each subscale for parents reporting their children's general health with higher categories (i.e., "excellent," "very good," "good," "fair," and "poor") $(p<0.001)$ and for children with a higher self-perception of their oral health $(p<0.001)$. Discriminant validity presented that the quality of life was better for school children with a satisfactory oral health status than for school children who had an oral health problem. In multiple linear regression analysis, a significant association was found between a lower oral health-related quality of life and decayed permanent teeth, missing permanent teeth, decayed deciduous teeth, gingivitis, and malocclusion after adjusting for other confounding variables.

Conclusion: Myanmar COHIP-SF 19 questionnaires were successfully developed and were appropriate for subjectively evaluating the oral health condition of children in Myanmar. 


\section{Introduction}

Oral Health-Related Quality Of Life (OHRQoL) is derived from a multidimensional construct that reveals individuals' well-being when eating, sleeping, and engaging in social communication, their self-esteem, and their fulfillment with respect to oral health [1]. The OHRQoL provides important facts when measuring the treatment requirements of individuals and populations, creating clinical decisions, and assessing interventions, preventive programs and services for oral health care [2].

Dental diseases and concomitant pain can greatly affect the daily life of a child by triggering a lack of sleep and by affecting the child's performance and quality of life $[3,4]$. To assess the condition of oral health, measuring the OHRQoL and the use of dental status indicators are necessary. In current years, various tools have been established and standardized for measuring the OHRQoL [5]. The short form of the Child Perception Questionnaires (CPQ11-14) [2] and the Child Oral Health Impact Profile (COHIP) [6] are the most commonly used questionnaires for evaluating OHRQoL in children [7].

Broder et al [6] developed COHIP to measure children's OHRQoL. It comprises 34 questions with five subscales: oral health, functional well-being, socioemotional well-being, school environment, and self-image. This instrument has good psychometric properties. The COHIP has been translated into many languages and its validity and reliability have been confirmed in cross-cultural adaptations [8,9]. For the purposes of need assessment and clinical research, the original COHIP was reduced to COHIP-SF 19 [10]. This reduced form contains 19 questions with three subscales: Oral Health $(\mathrm{OH})$ subscale, Functional Well-Being (FWB) subscale, and Socio Emotional Well-Being (SEWB) subscale. This shortened form maintains its strong reliability and validity properties.

The psychometric properties of the tools for assembling social data are influenced by the linguistic and cultural background where they are applied to collect data [11]. Hence, it is crucial to test the psychometric properties of the instruments, for assessing many further social or attitudinal measures before this instruments are used in a new circumstances [12]. The original version of COHIP-SF 19 has been translated into Chinese [13], Arabic [14], and Portuguese [15], and has good reliability and validity.

Sociodemographic data and the oral health condition of children substantively impact children's OHRQoL [16]. Among the OHRQoL instruments, Oral Impact on Daily Performance (OIDP) [17] and Oral Health Impact Profile (OHIP-14) [18] instruments have been validated in Myanmar [19]. However, no previous study has attempted to develop a Myanmar version of the COHIP-SF 19. Therefore, the purpose of the present study was to develop and measure the psychometric properties of the Myanmar COHIP-SF 19 and to identify the explanatory factors that impact the outcome of the OHRQoL among middle school children in Myanmar.

\section{Materials and methods}

This study was conducted in Yangon, Myanmar, and was targeted to 10 - to 11-year-old middle school children. All eligible school children, which consisted of grade $V$ school children $(n=569)$ from four public schools, were requested to participate. The inclusion criteria were school children who were without systemic disease or neuromuscular dysfunction, who obtained their parents' permission for participation, and who voluntarily participated in the study. Among these school children, 537 (boys $=251$ and girls=286) completed the questionnaires and clinical examinations. Ethics approval and consent to participate: Written consent was obtained 1 week before the study from the educational authorities, participants, and their parents or guardians. Ethical permission was obtained from the ethical committees of the Department of Medical Research in Myanmar (no. Ethics/DMR/2017/064), University of Dental Medicine, Yangon, and Tokyo Medical and Dental University (no. D2017-018). The research was done in full accordance with the Principle of Helsinki Declaration (World Medical Association, Ferney-Voltaire, France).

\section{Translation of the COHIP-SF 19}

Original English versions of the COHIP-SF 19 [10] were translated into Burmese, the major language spoken in Myanmar, and then back-translated. Expert panels confirmed the content and consistency. In a pilot study, the translated Myanmar versions were tested on 90 students. Based on the feedback, minor corrections were made so that the questionnaire would be suitable for school children. After 2 weeks, test-retest reliability testing was conducted on 43 school children.

\section{The COHIP-SF 19 questionnaires and scoring}

The COHIP-SF 19 Myanmar questionnaires consisted of 19 items that represent three conceptual subscales: (1) "Oral Health," $(\mathrm{OH})$ which consisted of five negatively worded questionnaires to evaluate specific oral health symptoms; (2) "Functional Well-Being," (FWB) which comprised four items of the children's capability to perform daily tasks; and (3) "Socioemotional Well-Being," (SEWB) which consisted of 10 items (eight items for peer interaction modes status and two items for positive self-image feelings). Answers to the 2 positively worded questionnaires were noted on a five-point Likert scale: $0=$ Never, 1 = Almost never, 2 = Sometimes, 3 = Fairly often, and $4=$ Almost all of the time. The total scores of the 17 negatively worded questionnaires was reversed, and the overall COHIPSF scores ranged from 0 to 76 . Higher scores reflected a better OHRQoL.

\section{Questionnaires}

Burmese was used for the assessment of all questionnaires. School children were requested to response questions about condition of their oral health by using a single-item global oral health rating, which had five possible responses (i.e., excellent, very good, good, fair, and poor [20,21]; the frequency of dental visits in the last 12 months and the reasons for a dental visit [22]. These standardized questionnaires were translated into Burmese (Myanmar language) and have previously been validated [23]. The parents of the participants gave information about their occupation [23]. Parents perception of their child's general health was measured with a single-item global health rating with answers ranging from " 1 " to " 5 "; higher scores indicated better general health [10].

\section{Clinical examination}

In the oral examination, one dentist assessed the school children's dental caries status (by using [DMFT] index), based on the guidelines of the World Health Organization (Geneva, Switzerland); oral hygiene status, based on the simplified oral hygiene index [24]; and gingival condition of 12 anterior teeth, based on the Papillary, Marginal, Attached gingiva (PMA) index (i.e., papillary, marginal, attached gingiva) [25]. The malocclu- 
sion status was measured using the Dental Aesthetic Index (DAI) [26]. A DAl score $>25$ indicated the presence of malocclusion. Intraexaminer agreement value was defined as "excellent" for dental caries (0.90) and as "good" for OHI-S (0.76), PMA (0.77) and DAI (0.70).

\section{Statistical analysis}

The internal consistency of the total COHIP-SF 19 score and three subscales score were quantified by means of the Cronbach's alpha value. The Intra class Correlation Coefficient (ICC) was used to calculate test-retest reliability. The KolmogorovSmirnov test was used for the distribution of the scores of the total COHIP-SF 19 and its subscales. Descriptive statistics of the study variables were calculated, based on sex. For the analysis, questionnaires regarding dental visits were categorized into two groups (i.e., "yes" group and "no" group). Categorical variables were defined and calculated by using the chi-square test. Mean differences among the continuous variables were analyzed with the Mann-Whitney $U$ test and the Kruskal-Wallis test. Concurrent validity was assessed by analyzing the total COHIP-SF and its subscales with the parents' observation of their child's general health and self-perception of oral health. Discriminant validity was further explored by using partial Spearman correlation to examine the relationship between the clinical index and the total COHIP-SF 19 scores, after adjusting for sex. Multiple linear regression tests were used to estimate the relationship between the dependent variable total COHIP-SF 19 scores with the independent variables such as: sex; decayed Permanent Teeth (DT) (“0" or " $\geq 1$ "); missing permanent teeth (MT) (“0" or " $\geq 1$ "); FT ("0" or " $\geq 1$ "); dt ("0" or " $\geq 1$ "); OHI-S score (Low score of $<1.50$ or High score of $\geq 1.50$ ); and PMA score (Low score of $\leq 17.0$ or high score of $>17.0$ ); DAl (normal occlusion [ $\leq 25=$ " 0 "] or malocclusion [ $>25=$ "1"]). Statistical Program of Social Science, version 21 (IBM, Tokyo, Japan) was used for data analysis.
The $p$ value for significant level of all results was set at 0.05 .

Results

\section{Reliability}

Cronbach's alpha value for the total COHIP-SF 19 score was 0.81 and for the three subscales was 0.61 for $\mathrm{OH}, 0.60$ for FWB, and 0.71 for SEWB. With regard to the test-retest reliability, the ICCs were 0.86, 0.63, 0.67, and 0.83 for the total COHIP-SF 19 scores, $\mathrm{OH}$ subscale, FWB, and SEWB, respectively.

Table 1 presents the data on dental visit experience and oral health status of the children, based on sex. Boys and girls were not significantly different with regard to dental visit experience, reasons for a dental visit, and oral health status, but they did differ with regard to gingivitis (based on the PMA index findings) and oral hygiene status (based on the OHI-S findings). The mean number of gingival inflammation sites was significantly lower in girls than in boys ( $15.5 \pm 5.6$ sites vs. $17.1 \pm 5.1$ sites; $p<$ $0.001)$. The OHI-S scores were significantly higher for boys (1.59 $\pm 0.62)$ than for girls $(1.31 \pm 0.54)(p<0.001)$.

The association between the scores of COHIP-SF 19 and the sociodemographic variables and dental visit experience is presented in Table 2. The scores of COHIP-SF 19 were not significantly related with the father's occupation, mother's occupation, or dental visit behavior. Scores for the total COHIP-SF 19, oral health, and functional well-being of the girls were significantly higher than in boys $(p=0.003, p<0.001$, and $p=0.024$, respectively).

\section{Validity}

The results of the concurrent validity of the COHIP-SF 19 with the parents' report of their children's general health status and the school children's self-perception of their oral health are

Table 1: Dental visit experience and oral health status

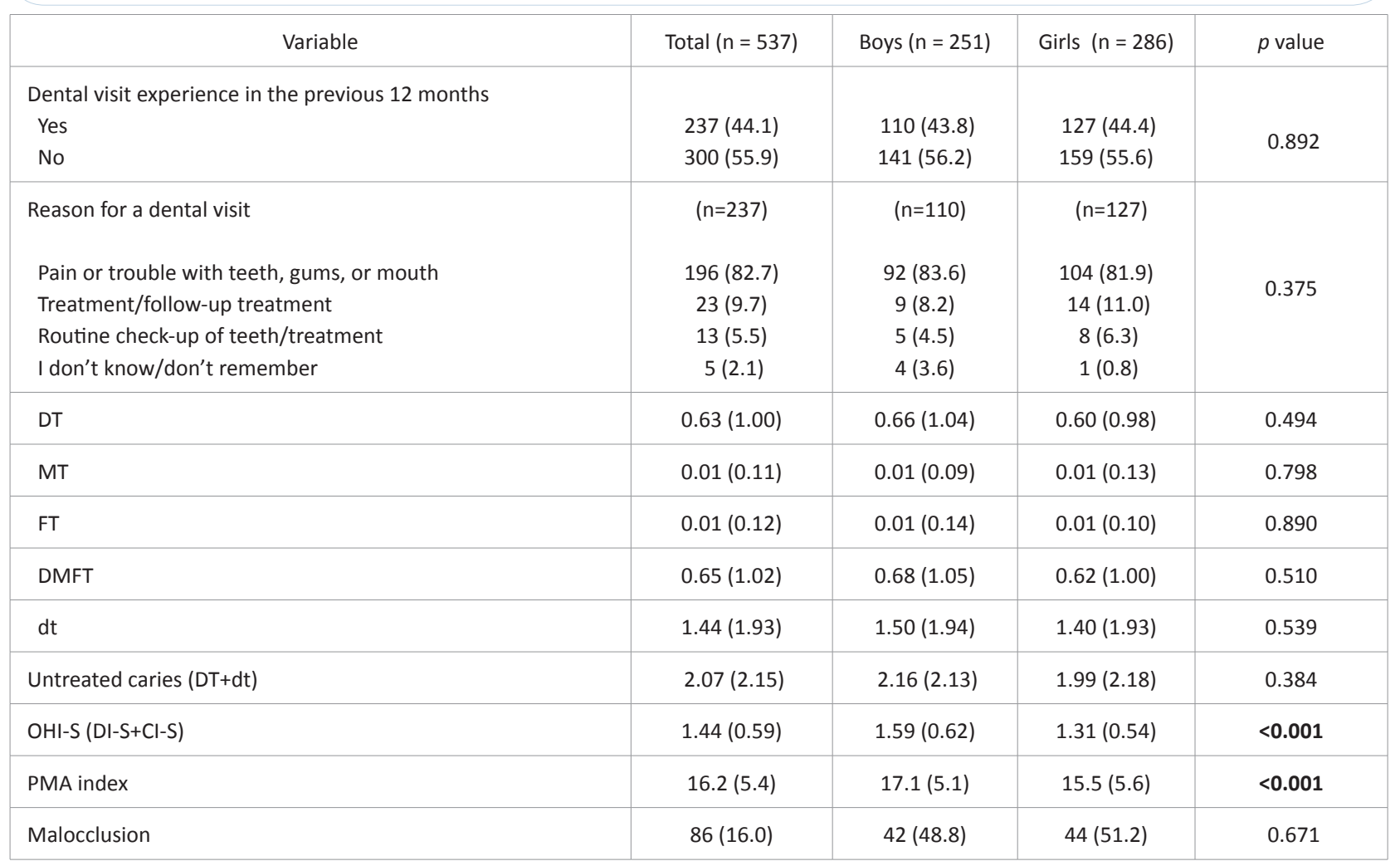

Note: The data are presented as the $\mathrm{n}(\%)$ or as the mean (SD) 
shown in Table 3. Discriminant validity was evaluated between clinical indexes and the total COHIP-SF 19 scores and its subscales, after controlling for sex (Table 4).

The number of active carious lesions (i.e., DT+dt) was negatively associated with the total COHIP-SF 19 and its three subscales $(p<0.001)$. Higher OHI-S scores among the school children had a significant impact on total COHIP-SF $19(p<0.001)$, $\mathrm{OH}(p<0.001)$, FWB $(p=0.005)$, and SEWB $(p=0.001)$. Moreover, the total COHIP-SF scores and three subscales were significantly lower for children with a greater level of gingival inflammation (based on the PMA index) $(p<0.001)$. However, their relationship was weak.

Table 5 presents the results of multiple linear regression between the total COHIP-SF 19 scores and potential explanatory variables. Significant predictors for lower COHIP-SF 19 scores were DT, MT, dt, gingivitis (based on the PMA index score), and malocclusion.

Table 2: Relationship between the COHIP-SF 19 scores and the sociodemographic variables and dental visit behavior

\begin{tabular}{|c|c|c|c|c|c|}
\hline Variable & N & $\mathrm{OH}$ & FWB & SEWB & Total COHIP-SF 19 \\
\hline \multicolumn{6}{|l|}{ Sex } \\
\hline Total & 537 & $13.73(3.14)$ & $12.99(2.46)$ & $31.79(5.18)$ & $58.45(8.82)$ \\
\hline Boys & 251 & $13.16(3.09)$ & $12.73(2.36)$ & $31.35(5.31)$ & $57.23(8.63)$ \\
\hline Girls & 286 & $14.23(3.12)$ & $13.21(2.53)$ & $32.17(5.04)$ & $59.53(8.86)$ \\
\hline$p$ value & & $<0.001$ & 0.024 & 0.069 & 0.003 \\
\hline \multicolumn{6}{|l|}{ Father's occupation } \\
\hline Government worker & 44 & $14.30(2.94)$ & $13.11(2.17)$ & $32.68(5.62)$ & $60.07(8.81)$ \\
\hline Professional & 34 & $14.76(2.63)$ & $13.68(2.27)$ & $32.38(5.47)$ & $60.82(7.91)$ \\
\hline Unskilled worker & 399 & $13.53(3.20)$ & $12.92(2.47)$ & $31.46(5.10)$ & $57.84(8.84)$ \\
\hline Merchant/seller & 40 & $14.28(2.75)$ & $13.20(2.34)$ & $33.75(4.66)$ & $61.23(8.04)$ \\
\hline Farmer & 12 & $12.83(3.86)$ & $12.00(3.49)$ & $32.08(5.50)$ & $56.92(9.81)$ \\
\hline Dependent/unemployed & 8 & $15.00(2.93)$ & $13.13(3.23)$ & $30.63(6.05)$ & $58.75(10.69)$ \\
\hline$p$ value & & 0.087 & 0.482 & 0.054 & 0.072 \\
\hline \multicolumn{6}{|l|}{ Mother's occupation } \\
\hline Government worker & 27 & $14.37(2.02)$ & $13.26(1.85)$ & $32.70(4.22)$ & $60.33(5.73)$ \\
\hline Professional & 5 & $15.60(1.52)$ & $13.40(1.34)$ & $32.00(4.85)$ & $58.80(8.90)$ \\
\hline Unskilled worker & 178 & $13.37(3.14)$ & $12.65(2.53)$ & $31.60(5.05)$ & $57.59(8.84)$ \\
\hline Merchant/seller & 2 & $13.00(4.24)$ & $11.00(4.24)$ & $35.00(2.83)$ & $59.00(11.31)$ \\
\hline Farmer & 5 & $15.60(1.52)$ & $13.00(3.08)$ & $30.20(5.63)$ & $58.80(8.81)$ \\
\hline Dependent/unemployed & 320 & $13.86(3.22)$ & $13.16(2.46)$ & $31.82(5.34)$ & $58.76(9.02)$ \\
\hline$p$ value & & 0.230 & 0.275 & 0.825 & 0.731 \\
\hline \multicolumn{6}{|l|}{ Dental visit } \\
\hline Yes & 237 & $13.75(3.13)$ & $12.90(2.49)$ & $31.97(4.99)$ & $58.59(8.58)$ \\
\hline No & 300 & $13.72(3.16)$ & $13.05(2.44)$ & $31.64(5.33)$ & $58.34(9.02)$ \\
\hline$p$ value & & 0.230 & 0.443 & 0.685 & 0.983 \\
\hline
\end{tabular}

Note: The data are presented as the mean (SD). SD: Standard Deviation; OH: Oral Health; FWB: Functional well-being; SEWB: Socioemotional well-being. 
Table 3: Concurrent validity of the COHIP-SF 19 scores versus general health and various oral health factors

\begin{tabular}{|c|c|c|c|c|c|c|}
\hline \multicolumn{2}{|c|}{ Variable } & \multirow{2}{*}{$\frac{N}{66}$} & \multirow{2}{*}{$\begin{array}{c}\mathrm{OH} \\
15.77(2.20)\end{array}$} & \multirow{2}{*}{$\begin{array}{c}\text { FWB } \\
14.08(1.74)\end{array}$} & \multirow{2}{*}{$\begin{array}{c}\text { SEWB } \\
36.61(3.66)\end{array}$} & \multirow{2}{*}{$\begin{array}{c}\text { Total COHIP-SF } 19 \\
66.30(5.57)\end{array}$} \\
\hline \multirow{5}{*}{$\begin{array}{l}\text { Parents perception } \\
\text { of a child's general } \\
\text { health }\end{array}$} & Excellent & & & & & \\
\hline & Very good & 120 & $15.33(2.64)$ & $14.12(1.82)$ & $34.43(4.03)$ & $63.88(6.53)$ \\
\hline & Good & 185 & $13.74(2.70)$ & $13.01(2.27)$ & $31.91(3.54)$ & $58.63(6.13)$ \\
\hline & Fair & 156 & $11.89(3.05)$ & $11.78(2.63)$ & $28.01(5.01)$ & $51.58(8.12)$ \\
\hline & Poor & 10 & $9.70(3.20)$ & $10.70(3.65)$ & $24.90(6.31)$ & $45.30(9.64)$ \\
\hline$p$ value & & & $<0.001$ & $<0.001$ & $<0.001$ & $<0.001$ \\
\hline \multirow{5}{*}{$\begin{array}{l}\text { Self-perception of } \\
\text { oral health }\end{array}$} & Excellent & 41 & $15.98(2.75)$ & $14.66(1.15)$ & $37.68(3.16)$ & $68.32(5.26)$ \\
\hline & Very good & 91 & $16.41(1.87)$ & $14.71(1.54)$ & $36.79(3.14)$ & $67.80(4.45)$ \\
\hline & Good & 153 & $14.53(2.30)$ & $13.69(1.75)$ & $32.42(3.33)$ & $60.62(4.75)$ \\
\hline & Fair & 179 & $12.72(2.70)$ & $12.32(2.01)$ & $30.07(3.71)$ & $55.02(5.12)$ \\
\hline & Poor & 73 & $9.96(2.34)$ & $10.07(2.97)$ & $25.12(4.45)$ & 45.14 (6.44) \\
\hline$p$ value & & & $<0.001$ & $<0.001$ & $<0.001$ & $<0.001$ \\
\hline \multirow[t]{2}{*}{ Malocclusion } & Normal & 451 & $13.76(3.11)$ & $13.08(2.36)$ & $32.12(4.96)$ & $58.91(8.52)$ \\
\hline & malocclusion & 86 & $13.56(3.31)$ & $12.48(2.91)$ & $30.07(5.94)$ & 56.05 (9.92) \\
\hline$p$ value & & & 0.576 & 0.037 & 0.001 & 0.006 \\
\hline
\end{tabular}

Note: The data are presented as the mean (SD). COHIP-SF 19, Child Oral Health Impact Profile-Short Form 19; SD: standard deviation; OH: Oral Health; FWB: Functional well-being; SEWB: Socioemotional well-being.

Table 4: Discriminant validity: correlation between oral health status and the COHIP-SF 19 and three subscales

\begin{tabular}{|c|c|c|c|c|c|c|}
\hline \multirow{2}{*}{$\begin{array}{c}\text { Variable } \\
\text { mean (SD) }\end{array}$} & \multicolumn{2}{|c|}{ Active caries (DT+dt) } & \multicolumn{2}{|c|}{ OHI-S (DI+CI) } & \multicolumn{2}{|c|}{ Gingivitis (PMA) } \\
\cline { 2 - 6 } & $r_{s}$ & $p$ value & $r_{s}$ & $p$ value & $r_{s}$ & $p$ value \\
\hline Oral health & -0.349 & $<0.001$ & -0.207 & $<0.001$ & -0.170 & $<0.001$ \\
\hline Functional well-being & -0.242 & $<0.001$ & -0.137 & $\mathbf{0 . 0 0 5}$ & -0.167 & $<0.001$ \\
\hline Socioemotional well-being & -0.247 & $<0.001$ & -0.200 & $\mathbf{0 . 0 0 1}$ & -0.220 & $<0.001$ \\
\hline Total COHIP-SF 19 & -0.336 & $<0.001$ & 0.227 & $<0.001$ & -0.236 & $<0.001$ \\
\hline
\end{tabular}

Note: Partial Spearman correlation analysis was conducted, after adjusting for sex.

Table 5: Multiple linear regression analysis between the clinical index and the total COHIP-SF 19 score

\begin{tabular}{|l|c|c|c|}
\hline \multirow{2}{*}{ Independent variable } & \multicolumn{3}{|c|}{ COHIP-SF 19 } \\
\cline { 2 - 4 } & $\mathrm{B}$ & Standard error & $p$ value \\
\hline Sex & 1.304 & 0.713 & 0.068 \\
\hline DT & -5.663 & 0.734 & $<0.001$ \\
\hline MT & -13.860 & 4.138 & 0.001 \\
\hline FT & -2.375 & 3.682 & 0.519 \\
\hline dt & -2.615 & 0.693 & $<0.001$ \\
\hline OHI-S (DI+CI) & -1.212 & 0.784 & 0.149 \\
\hline PMA index & -1.609 & 0.778 & $\mathbf{0 . 0 3 2}$ \\
\hline Malocclusion & -3.257 & 0.940 & $\mathbf{0 . 0 0 1}$ \\
\hline
\end{tabular}

Note: The scores for the independent variables are as follows:

For sex, boy: 0 and girl: 1 ; for DT, caries-free permanent teeth: 0 and $\geq 1$ caries in permanent teeth: 1 ; for MT, no missing permanent teeth: 0 and $\geq 1$ missing permanent teeth: 1 ; for FT, no filled permanent teeth: 0 and $\geq 1$ filled permanent teeth: 1 ; for $\mathrm{dt}$, caries-free primary teeth: 0 and $\geq 1$ caries in the primary teeth: 1 ; for $\mathrm{OHI}-\mathrm{S}$, Low score of $<1.50=$ 0 and High score of $\geq 1.50$; for PMA, Low score of $\leq 17.0$ and High score of >17.0; and for malocclusion, "no": 0 and "yes": 1 . B: regression coefficient 


\section{Discussion}

The objectives of the current study was to evaluate the psychometric properties of a Myanmar COHIP-SF 19 and to identify the explanatory factors that impact the COHIP-SF 19. The outcomes of this study showed that the Myanmar COHIP-SF 19 was successfully developed and cross-culturally adapted, and it had acceptable psychometric properties for Myanmar middle school children. The scores of the Myanmar COHIP-SF 19 demonstrated good validity and acceptable reliability, which support its use for 10- to 11-year-old children. For that reason, the Myanmar version of the COHIP-SF 19 can be used in crosscultural comparisons between the Myanmar version and other language versions.

The Cronbach's alpha value for total COHIP-SF 19 scores showed good reliability, which was consistent with that of the original English COHIP-SF 19 version [10], and the Chinese [13], Arabic [14], and Brazilian [15], versions.

The socioemotional subscale exhibited an acceptable level. Cronbach's alpha values for the $\mathrm{OH}$ and the FWB were lower than those of the SEWB. These results corroborated previous findings in the Chinese version [13] and the Arabic version [14]. Oral health and functional well-being have few items, which may have an effect on Cronbach's alpha value $[27,28]$. The ICC value of the total COHIP-SF 19 score indicated excellent reliability and that of each subscale showed moderate reliability.

Government workers and professionals in Myanmar have a higher socioeconomic status than do individuals who work in other occupations [23]. However, in the current study, a parent's occupation did not have an effect on the OHRQoL of their children. The findings of two studies contrast with those of our study: one team in Brazil reported that the children of unemployed fathers were at a greater risk of having a poor OHRQoL than were children who had employed fathers [16], and another team in Brazil reported that maternal employment was a significant predictor of their children's OHRQoL-employed mothers reported a poorer OHRQoL for their children than mothers who were not working [29]. In the current study, dental visit experience was not a significant factor for OHRQoL. In Norway, regular dental attendees reported a better OHRQoL than did irregular attendees [30]. An explanation for the findings of our study could be that, among children who previously had a dental visit, $82.7 \%$ had done so because of problems with their teeth or gums and only $5.5 \%$ had regular dental checkups. Therefore, regular dental visits are important for improving the OHRQoL.

The concurrent validity test revealed higher total COHIP-SF 19 scores and higher scores for each subscale among parents reporting higher categories (i.e., excellent, very good etc) for their children's general health $(p<0.001)$ and among children with a higher self-perception of their oral health $(p<0.001)$. The findings of this testing corroborate those of other reports $[10,13,14]$.

The school children who had malocclusion (based on DAI index) had a lower OHRQoL, except for the oral health subscale. Previous studies that were conducted in the United States [10], China [13], and Zambia [31] confirm these findings. The result differs from that of other studies conducted in Brazil [32] and Japan [33].

The mean scores of the Myanmar COHIP-SF 19 were low, compared to those reported in studies conducted in China [13] and Libya [14]. School children in the current study had expe- rienced dental caries in their permanent and deciduous teeth, poor oral hygiene, exfoliation of primary teeth, and space because of unerupted permanent teeth caused by mixed dentition, all of which may have affected their OHRQoL. In addition, discriminant validity testing showed a significant relationship between active carious lesions, oral hygiene status, and gingivitis with lower OHRQoL. However, their association was weak.

The outcome of the discriminant validity testing demonstrated that COHIP-SF 19 could be used to categorize between children with and without oral health problems. The quality of life was better for students with a satisfactory oral health status than for students with oral health problems $[8,19,34]$.

In this study, the mean scores of the total COHIP-SF 19, OH, and FWB were significantly higher in girls than in boys. This finding agrees with that of a previous study [13]. The reason for sex differences in OHRQoL, except for socioemotional well-being, may be because the oral hygiene status and gingivitis condition of the girls were significantly lower than in boys. However, the socioemotional well-being subscale score was higher, but not significantly so, among boys than among girls. This finding may be because, compared to girls, boys are probably less selfconscious than girls regarding facial appearance and emotion. The present study found a significant association between DT, MT, decayed deciduous teeth, gingivitis, malocclusion, and lower OHRQoL, after adjusting by using multiple linear regression analysis. However, the correlation of sex with OHRQoL was not significantly different. This result is reliable with the findings of the aforementioned study conducted in Portugal [35].

One of the limitations of the present study was that the participants were obtained from only one region in Myanmar. For that reason, the findings of the current study may have restricted generalizability to other areas in the nation. Age-related variation was not explored because of the target population was only 10-11 years old. Further intervention studies with various age groups are needed to evaluate the outcome measure and applicability of the Myanmar COHIP-SF 19.

\section{Conclusions}

Poor oral health and malocclusion are significantly associated with a lower OHRQoL. The findings of the current study support that the Myanmar version of COHIP-SF 19 questionnaires has good psychometric properties. Therefore, this instrument was successfully developed and is appropriate for the subjective evaluation of the oral health condition of children in Myanmar.

\section{Acknowledgements}

We wish to express my heartfelt thanks to the school authorities and school children who participated in the study. We thanks to FUTOKUKAI Foundation (Japan), and Department of Oral Health Promotion at Tokyo Medical and Dental University for their financial support.

\section{References}

1. U.S. Department of Health and Human Services. Oral health in America: a report of the Surgeon General. Rockville, MD: U.S. Department of Health and Human Services, National Institute of Dental and Craniofacial Research, National Institutes of Health; 2000; 7 .

2. Jokovic A, Locker D, Guyatt G. Short forms of the Child Perceptions Questionnaire for 11-14-year-old children (CPQ11-14): development and initial evaluation. Health Qual Life Outcomes. 2006; $4: 4$. 
3. Nuttall NM, Steele JG, Evans D, Chadwick B, Morris AJ. et al. The reported impact of oral condition on children in the United Kingdom, 2003. Br Dent J. 2006; 200: 551-555.

4. Bali Declaration-Good Oral Health as Investment in Children's Future. The 7th Asian Conference of Oral Health Promotion for School Children (ACOHPSC). 2013.

5. Sischo L, Broder HL. Oral health-related quality of life: what, why, how, and future implications. J Dent Res. 2011; 90: 12641270 .

6. Broder HL, Wilson-Genderson M. Reliability and convergent and discriminant validity of the Child Oral Health Impact Profile (COHIP Child's version). Community Dent Oral Epidemiol. 2007; 35: 20-31.

7. Foster Page L, Gilchrist F, Broder HL, Clark E, Thomson WM. A comparison of three child OHRQoL measures. Dent J (Basel). 2019; 7.

8. El Osta N, Pichot H, Soulier-Peigue D, Hennequin M, TubertJeannin S. Validation of the child oral health impact profile (COHIP) French questionnaire among 12 years-old children in New Caledonia. Health Qual Life Outcomes. 2015; 13: 176.

9. Ahn YS, Kim HY, Hong SM, Patton LL, Kim JH, et al. Validation of a Korean version of the Child Oral Health Impact Profile (COHIP) among 8- to 15-year-old school children. Int J Paediatr Dent. 2012; 22: 292-301.

10. Broder $\mathrm{HL}$, Wilson-Genderson M, Sischo L. Reliability and validity testing for the Child Oral Health Impact Profile-Reduced (COHIP-SF 19). J Public Health Dent. 2012; 72: 302-312.

11. Nyklícek I. Research methods in health. J Psychophysiol. 2000; 14: 126-128.

12. Robinson PG, Gibson B, Khan FA, Birnbaum W. Validity of two oral health-related quality of life measures. Community Dent Oral Epidemiol. 2003; 31: 90-99.

13. Li C, Xia B, Wang Y, Guan X, Yuan J. et al. Translation and psychometric properties of the Chinese (Mandarin) version of the Child Oral Health Impact Profile-Short Form 19 (COHIP-SF 19) for school-age children. Health Qual Life Outcomes. 2014; 12: 169.

14. Arheiam AA, Baker SR, Ballo L, Elareibi I, Fakron S, et al. The development and psychometric properties of the Arabic version of the child oral health impact profile-short form (COHIP- SF 19). Health Qual Life Outcomes. 2017; 15: 218.

15. Martins LGT, Parma GOC, Cristiano D, Possamai CF, Sônego FGF, et al. Cross-cultural adaptation and validation of the COHIP-SF19 to be used in Brazil. Pesquisa Brasileira em Odontopediatria e Clínica Integrada. 2018; 18: 1-13.

16. Piovesan C, Antunes JL, Guedes RS, Ardenghi TM. Impact of socioeconomic and clinical factors on child oral health-related quality of life (COHRQoL). Qual Life Res. 2010; 19: 1359-1366.

17. Adulyanon $S$, Sheiham A. Oral impacts on daily performances. In: Slade GD, editor. Measuring oral health and quality of life. vol. 151. Chapel Hill, NC: University of North Carolina, Dental Ecology. 1997; 150-161.

18. Slade GD, Spencer AJ. Development and evaluation of the Oral Health Impact Profile. Community Dent Health. 1994; 11: 3-11.

19. Soe KK, Gelbier S, Robinson PG. Reliability and validity of two oral health related quality of life measures in Myanmar adolescents. Community Dent Health. 2004; 21: 306-311.
20. Thomson WM, Mejia GC, Broadbent JM, Poulton R. Construct validity of Locker's global oral health item. J Dent Res. 2012; 91: 1038-1042.

21. Slade GD. Oral health-related quality of life. Carol Stream, IL: Quintessence Publishing Co, Inc; 2002.

22. World Health Organization (WHO). Oral health surveys: basic methods. 5th edition. Geneva, Switzerland: WHO. 2013.

23. Kyaw Myint ZC, Zaitsu T, Oshiro A, Ueno M, Soe KK, et al. Risk indicators of dental caries and gingivitis among 10-11-year-old students in Yangon, Myanmar. Int Den J 2019. doi: 10.1111/ idj.12537

24. Greene JC, Vermillion JR. The Simplified Oral Hygiene Index. J Am Dent Assoc. 1964; 68: 7-13.

25. Massler M, Schour I. Epidemiology of gingivitis in children. J Am Dent Assoc. 1952; 45: 319-324.

26. Jenny J, Cons NC. Establishing malocclusion severity levels on the Dental Aesthetic Index (DAI) scale. Aust Dent J. 1996; 41: 43-46.

27. Steiner DL. A checklist for evaluating the usefulness of rating scales. Can J Psychiatry. 1993; 38: 140-148.

28. Gliem JA, Gliem RR. Calculating, interpreting, and reporting Cronbach's alpha reliability coefficient for Likert-type scales. 2003 Midwest Research to Practice Conference in Adult, Continuing, and Community Education; Columbus, $\mathrm{OH}$ : Ohio State University; 2003; 82-88.

29. Peres KG, Peres MA, Araujo CL, Menezes AM, Hallal PC. Social and dental status along the life course and oral health impacts in adolescents: a population-based birth cohort. Health Qual Life Outcomes. 2009; 7: 95.

30. Dahl KE, Wang NJ, Skau I, Ohrn K. Oral health-related quality of life and associated factors in Norwegian adults. Acta Odontol Scand. 2011; 69: 208-214.

31. Anthony SN, Zimba K, Subramanian B. Impact of malocclusions on the oral health-related quality of life of early adolescents in Ndola, Zambia. Int J Dent. 2018; 2018: 7920973.

32. Barbosa TS, Tureli MC, Gaviao MB. Validity and reliability of the child perceptions questionnaires applied in Brazilian children. BMC Oral Health. 2009; 9: 13.

33. Yamada H, Miura H, Usui Y, Sato T. Development of the Japanese version of the Child Perceptions Questionnaire (CPQ 8-10_JP). Pediatr Dent J. 2013; 23: 86-90.

34. Abanto J, Tsakos G, Paiva SM, Carvalho TS, Raggio DP, et al. Impact of dental caries and trauma on quality of life among 5- to 6-year-old children: perceptions of parents and children. Community Dent Oral Epidemiol. 2014; 42: 385-394.

35. Rando GM, Jorge PK, Vitor LLR, Carrara CFC, Soares S. et al. Oral health-related quality of life of children with oral clefts and their families. J Appl Oral Sci. 2018; 26: 20170106. 